\title{
Synergistic effect of survivin-specific small interfering RNA and topotecan in renal cancer cells: Topotecan enhances liposome- mediated transfection by increasing cellular uptake
}

\author{
AKINORI SATO, KEIICHI ITO, TAKAKO ASANO, MAKOTO SUMITOMO, \\ TOMOHIKO ASANO and MASAMICHI HAYAKAWA \\ Department of Urology, National Defense Medical College, 3-2 Namiki, Tokorozawa, Saitama 359-8513, Japan
}

Received July 31, 2006; Accepted September 28, 2006

\begin{abstract}
Survivin, an inhibitor of apoptosis (IAP) protein detected in many tumors but not in most normal differentiated tissues, has been widely recognized as an attractive target for cancer therapy. We previously showed that survivin expression is associated with cell proliferation. Although liposome-mediated transfection of survivin-specific siRNA decreases survivin expression and cell proliferation, these effects are limited in part by the low efficiency of the transfection. In the present study we therefore investigated the possibility of better suppressing survivin expression and cell growth by using treatments combining survivin-specific siRNA and the topoisomerase I inhibitor topotecan. Survivin-specific siRNA and topotecan given simultaneously inhibited survivin expression and cell proliferation synergistically, but topotecan alone or topotecan and siRNA given metachronously did not alter survivin expression. We hypothesized that topotecan increases the efficiency of siRNA transfection by increasing cellular uptake, and we confirmed this hypothesis by using fluorescein-labeled siRNA. Combination therapy using survivin-specific siRNA and topotecan should thus show a synergistic effect due to increased cellular uptake of siRNA and offer an attractive approach for treatment of advanced renal cancer.
\end{abstract}

\section{Introduction}

Renal cancer accounts for 2-3\% of adult cancers and for $2 \%$ of all cancer-related deaths (1). Approximately half of the patients with renal cancer have metastases at the time of diagnosis or will develop them during the clinical course (2). The outcome of the treatment of advanced renal cancer is disappointing. Conventional cytotoxic chemotherapy and radiotherapy are largely ineffective (3), response rates to

Correspondence to: Dr Akinori Sato, Department of Urology, National Defense Medical College, 3-2 Namiki, Tokorozawa, Saitama 359-8513, Japan

E-mail: zenpaku@ndmc.ac.jp

Key words: renal cancer, survivin, topotecan, siRNA, lipofection, transfection efficiency interferon (IFN)- $\alpha$ and IFN- $\beta$ treatments range from 10 to $20 \%$ (4), and long-lasting responses to interleukin (IL)-2-based therapy are obtained in only $4-5 \%$ of the treated patients (5). Although there have been numerous attempts to increase these low response rates by using combination therapies, such as vinblastine and IFNs, the reported effects have not been dramatic $(6,7)$. Further improvement awaits the development of a more effective systemic therapy.

Survivin is one of the inhibitor of apoptosis (IAP) proteins detected in many tumors but is absent in most normal differentiated tissues (8). Unlike other IAPs, survivin contains a single BIR and lacks a carboxyl-terminal RING finger (8). Survivin is expressed in the G2-M phase in a cell cycledependent manner, and binds directly to mitotic spindle microtubules (9). It has also been shown to inhibit the processing of procaspases-3 and -7 and to specifically bind both active caspases (10). Survivin thus inhibits apoptotic stimuli and its expression is associated with carcinogenesis, tumor progression, and decreased survival (11). It has therefore been widely recognized as an attractive target for cancer therapy, and the suppression of survivin expression by antisense oligonucleotides or small interfering RNA (siRNA) is a promising approach to selective cancer therapy (12-14).

We have previously shown that survivin expression is associated with the proliferation of renal cancer cells, but their proliferation was not completely inhibited by survivinspecific siRNA (15). The transfection efficiency of liposomebased gene delivery is generally low and complete suppression of survivin expression is not always achieved. One might thus expect to achieve more thorough inhibition of survivin expression and cell proliferation by using combination therapies exploiting siRNA and chemotherapeutic agents.

In the present study we investigated the response of renal cancer cells to treatment with a combination with survivinspecific siRNA and the topoisomerase I inhibitor topotecan. We found a synergistic effect of survivin-specific siRNA and topotecan on the suppression of survivin expression and cell growth. We also found that topotecan enhanced liposomemediated transfection of siRNA by increasing cellular uptake. The synergistic effect of this combination treatment is thought to be due in part to topotecan increasing the cellular uptake of siRNA. Combining survivin-specific siRNA and topotecan is thus a promising approach to the treatment of advanced renal cancer. 


\section{Materials and methods}

Cell culture. A KU19-20 cell line, derived from human renal cancer, was established at Keio University. Cells were grown in RPMI-1640 medium containing 10\% fetal bovine serum and $0.3 \%$ penicillin-streptomycin (Invitrogen, Carlsbad, CA, USA).

Treatment with survivin-specific siRNA and topotecan. Cells were plated in 6-well (for Western blot analysis) or 96-well (for cell proliferation assay) culture plates one day before treatment so they would reach about $70 \%$ confluence. The survivinspecific siRNA or non-sense siRNA (Santa Cruz Biotechnology, Santa Cruz, CA, USA) (each $100 \mathrm{nM}$ ) was mixed with Lipofectamin 2000 (Invitrogen, Carlsbad, CA, USA) following the manufacturer's instruction and administered to the cells in the RPMI-1640 medium containing topotecan (Calbiochem, San Diego, CA, USA) at concentrations ranging from 0 to $1 \mu \mathrm{g} / \mathrm{ml}$. Cells were maintained in this medium for 24 or $48 \mathrm{~h}$ and then assayed for survivin suppression and cell proliferation.

For metachronous treatment, cells were first treated with survivin-specific siRNA for $24 \mathrm{~h}$ and then cultured in the medium with or without topotecan for another $24 \mathrm{~h}$ before being subjected to Western blot analysis.

Cell proliferation assay. Cells were plated in 96-well culture plates $24 \mathrm{~h}$ prior to treatment and then incubated under the indicated conditions for $48 \mathrm{~h}$ after which cell proliferation was determined using the MTT assay.

Western blot analysis. Samples were mixed with loading buffer, resolved by $12.5 \%$ SDS-PAGE, and transblotted onto nitrocellulose membranes. After blocking with 5\% dry skim milk, the membranes were incubated for an hour or overnight with anti-survivin rabbit polyclonal antibody (Alpha Diagnostic International, San Antonio, TX, USA) before being incubated with a biotinylated secondary goat anti-rabbit antibody (Bio-Rad Laboratories, Hercules, CA, USA) at room temperature. The membranes were then incubated for $1 \mathrm{~h}$ in streptavidin-biotinylated alkaline phosphatase complex (BioRad Laboratories) at room temperature. Immunoreactivity was detected using an alkaline phosphatase substrate kit (Bio-Rad Laboratories).

Cell cycle analysis. At indicated time intervals after treatment with survivin-specific siRNA and/or topotecan as described above, KU19-20 cells were washed with PBS and harvested by trypsinization. After they were resuspended in citrate buffer and stained with propidium iodide according to the method by Vindelov et al (16), they were analyzed by flow cytometry using the Cell Quest program for calculation of the different cell cycle phases.

Measurement of cellular uptake of siRNA. KU19-20 cells were transfected with fluorescein-labeled non-sense siRNA (Santa Cruz Biotechnology) as described above. They were cultured in the RPMI-1640 medium with or without topotecan $(0-1 \mu \mathrm{g} / \mathrm{ml})$ for $48 \mathrm{~h}$, washed with PBS, and harvested by trypsinization. Cellular uptake of fluorescein-labeled siRNA was measured by flow cytometry using the Cell Quest program.
A

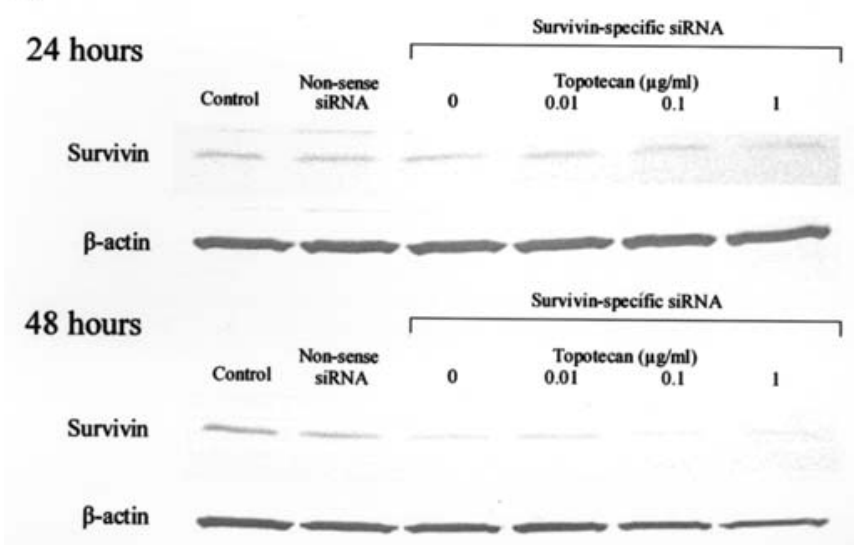

B

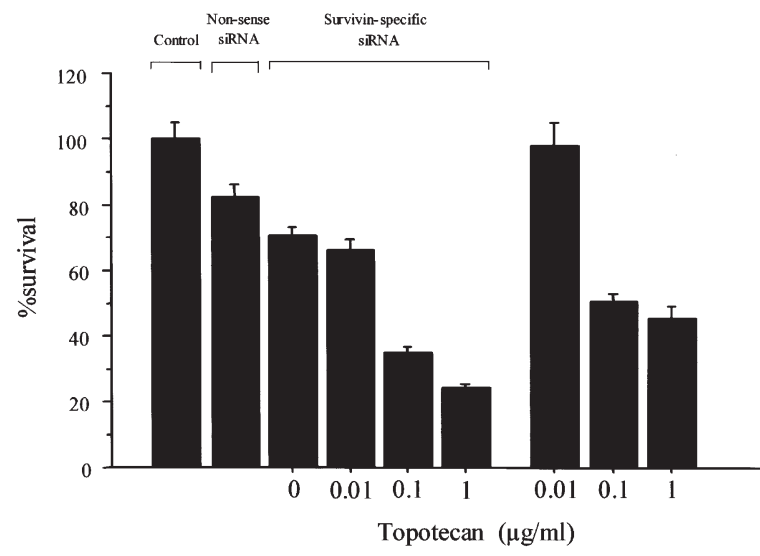

Figure 1. Inhibition of survivin expression and cell proliferation by simultaneous treatment with survivin-specific siRNA and topotecan. (A) Survivin Western blot analysis after 24- and 48-h treatment. Survivin expression was further suppressed by the simultaneous treatment with siRNA and topotecan in a dose-dependent manner. (B) Cell viability using MTT assay after 48-h treatment. Cell proliferation was synergistically inhibited by the combination of siRNA and topotecan.

\section{Results}

Successful uptake of siRNA under the conditions used in this study was confirmed in a previous study by using fluoresceinlabeled siRNA and an epifluorescence microscope (15). In the present study we found that a more thorough inhibition of survivin expression and cell proliferation could be obtained by treating the cells with both survivin-specific siRNA and topotecan. Simultaneous treatment with siRNA and topotecan for 24 or $48 \mathrm{~h}$ inhibited survivin expression more than did treatment with siRNA alone (Fig. 1A) and the 48-h combination treatment inhibited cell proliferation synergistically (Fig. 1B).

The results of the simultaneous treatment with survivinspecific siRNA and topotecan indicated that topotecan increased the efficiency of siRNA transfection and/or topotecan itself decreased the expression of survivin. To explore the mechanism of the topotecan-induced enhancement of the decline in survivin expression, we investigated the effects of metachronous treatment with survivin-specific siRNA and topotecan. If the enhancement were due to increased cellular uptake, it would not occur when siRNA and topotecan were administered metachronously. 
A

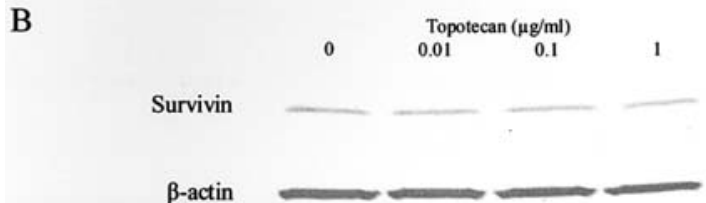

Figure 2. Effect of metachronous treatment with survivin-specific siRNA and topotecan or topotecan alone on survivin expression. (A) Survivin Western blot analysis after metachronous treatment with siRNA (24 h) and topotecan $(24 \mathrm{~h})$. Different from the simultaneous treatment, no further suppression of survivin was seen by the combination of siRNA and topotecan. (B) Survivin Western blot analysis $48 \mathrm{~h}$ after treatment with topotecan alone. Topotecan itself had no effect on survivin espression.

When the cells were treated first with survivin-specific siRNA for $24 \mathrm{~h}$ and then with topotecan for $24 \mathrm{~h}$ (metachronous treatment), no further suppression of survivin was observed (Fig. 2A). Administration of topotecan alone for $48 \mathrm{~h}$ also did not alter survivin expression (Fig. 2B). From these results we inferred that topotecan itself had no effect on survivin expression but enhanced the transfection of siRNA.

Cell cycle analysis comparing the treatment with topotecan alone and the simultaneous administration of survivin-specific siRNA and topotecan for $48 \mathrm{~h}$ showed that the combination treatment increased the sub-G1 fraction, which included apoptotic cells induced by the suppression of survivin, without markedly changing other cell cycle phases. This result supports the hypothesis that topotecan increases the survivin suppression due to siRNA. The effect of the 48-h treatment with topotecan on cell cycle varied with concentration. The treatment with $0.1 \mu \mathrm{g} / \mathrm{ml}$ topotecan led to accumulation of the S-phase fraction, whereas the treatments with 0.01 and $1 \mu \mathrm{g} / \mathrm{ml}$ topotecan increased the G1 fraction (Fig. 3).

To find out if topotecan increased transfection efficiency by increasing the cellular uptake of siRNA, we evaluated the
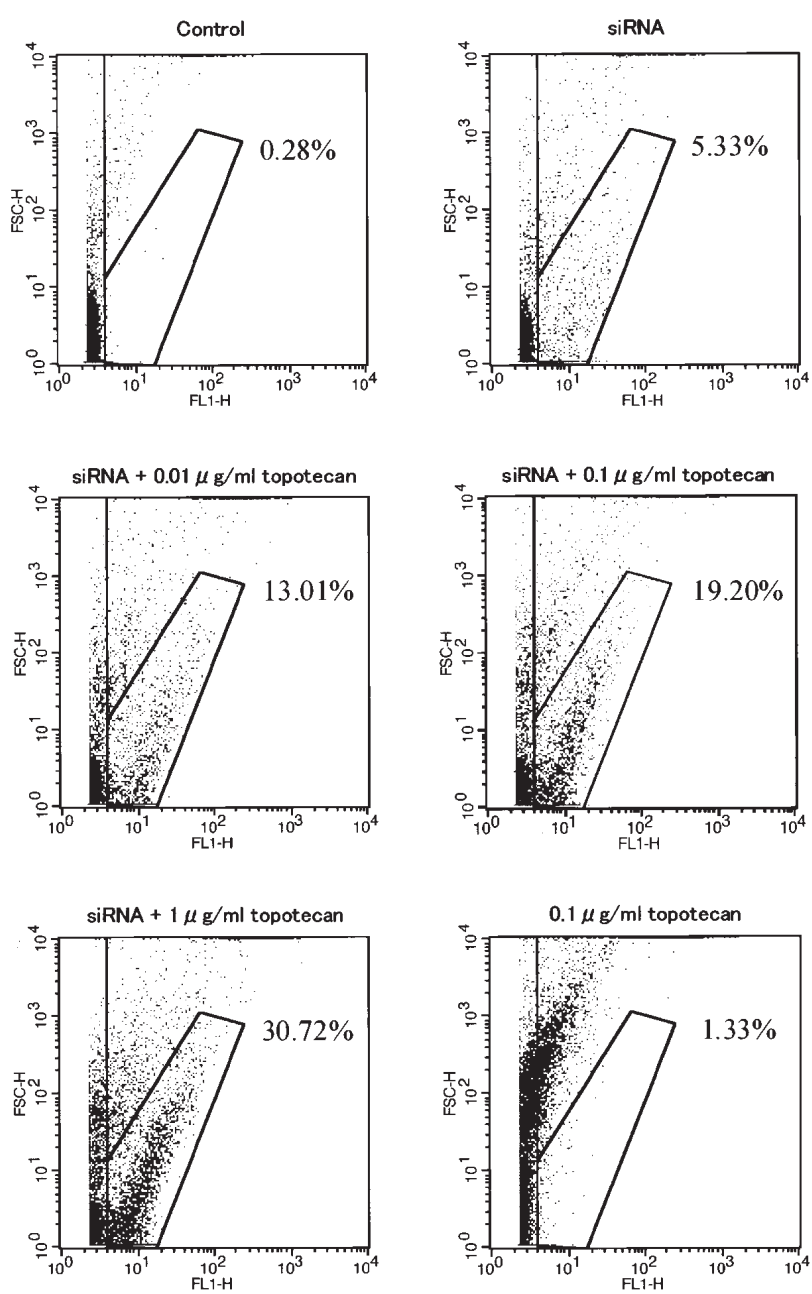

Figure 4. Analysis of cellular uptake of siRNA by flow cytometry $48 \mathrm{~h}$ after administration of fluorescein-labeled siRNA and topotecan. Percentage of the cells which incorporated liposome-fluorescein-labeled siRNA complex is measured. By the simultaneous administration of siRNA and topotecan, cellular uptake of siRNA was increased in a topotecan dose-dependent manner.

changes in the cellular uptake of siRNA by using fluoresceinlabeled non-sense siRNA. Because the cellular uptake of siRNA was increased by the simultaneous administration of topotecan (Fig. 4), we therefore inferred that one mechanism

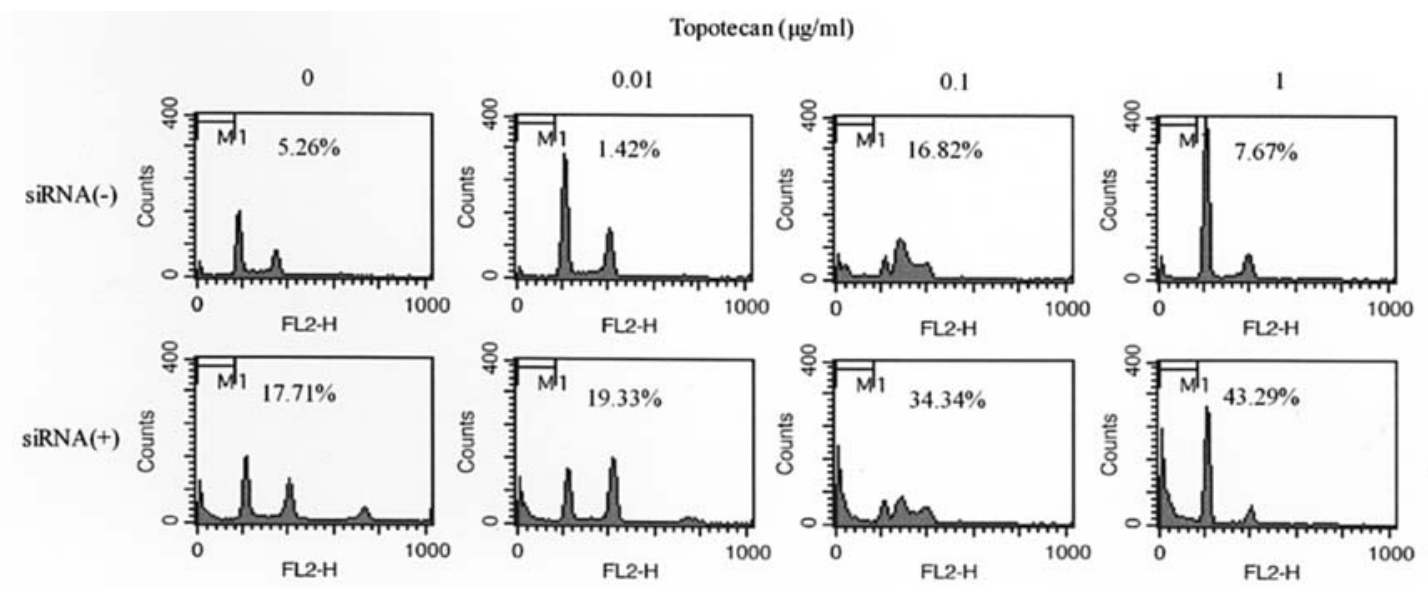

Figure 3. Cell cycle analysis $48 \mathrm{~h}$ after treatment with survivin-specific siRNA and/or topotecan. Percentage of the cells in the sub-G1 phase is shown. The simultaneous treatment with siRNA and topotecan increased cells in the sub-G1 fraction in a dose-dependent manner. 

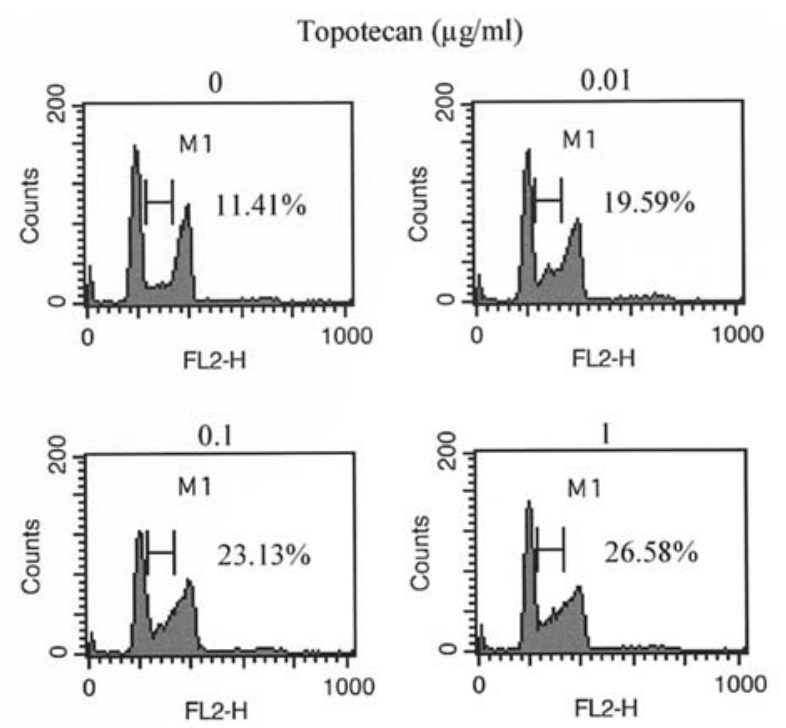

Figure 5. Cell cycle analysis $6 \mathrm{~h}$ after treatment with topotecan. Percentage of the cells in the S-phase is measured. The S-phase fraction was increased by topotecan in a dose-dependent manner.

of the synergistic effect of survivin-specific siRNA and topotecan is topotecan-increased uptake of siRNA.

Thinking that topotecan-induced changes in the cell cycle in the early phase of transfection would shed light on the mechanism of the increased cellular uptake of siRNA, we evaluated the change in the cell cycle after 6-h treatment with topotecan. Cell cycle analysis revealed that the 6-h treatment with topotecan increased the S-phase fraction in a dosedependent manner (Fig. 5). The accumulation in the S-phase may be one of the mechanisms of increased cellular uptake of siRNA.

\section{Discussion}

Survivin, which is highly expressed in cancer cells and undetectable in terminally differentiated tissues, may play an important role in carcinogenesis (8). Its expression in renal cancer cells is associated with cell proliferation (15), and survivin may be a promising target for gene therapy in advanced renal cancer.

RNA interference (RNAi) is an evolutionarily ancient mechanism of gene regulation in eukaryotes and has become a powerful tool for probing gene function. The general mechanism of RNAi involves the cleavage of double-stranded RNA (dsRNA) to short siRNAs. This processing is catalyzed by Dicer, a highly conserved dsRNA-specific endonuclease that is a member of the RNase III family. The siRNAs are then incorporated into the RNA-induced silencing complex (RISC) and guide the destruction or repression of complementary mRNAs (17). Recent studies have found that knockdown of survivin expression by siRNA decreases cell survival in human sarcoma (14) and renal cancer (15). Furthermore, liposome-mediated systemic delivery of siRNA has been shown to inhibit exogenous and endogenous gene expression in vivo (18). Suppression of survivin expression by siRNA would thus seem to be a promising means of selective cancer therapy.
In the present study survivin expression was inhibited by the liposome-mediated transfection of survivin-specific siRNA, but cell proliferation was not completely suppressed. Similar results have been obtained in other studies using antisense oligonucleotides or siRNA $(13-15,19)$. Although survivin mRNA has been reported to be one of the four most highly expressed transcripts in tumor cells (20) and would thus code for one of the major regulatory proteins in cancer cells, it is natural that other molecules and different signaling pathways also play important roles in the proliferation of cancer cells. Also, because transfection efficiency usually differs between cell types and cell conditions, survivin expression would not always be suppressed completely. We postulated that cancer cell damage could be enhanced by combining the use of survivin-specific siRNA with the use of chemotherapeutic agents.

Topotecan is a topoisomerase I inhibitor whose effectiveness in patients with advanced renal cancer has so far been assessed only in one small phase II trial (21). Ramp et al have however shown not only that clinically relevant doses of topotecan are effective in human renal cancer cell lines but also that in these lines topotecan is more effective than 5-fluorouracil (22). The present study showed that topotecan is also effective in another line of human renal cancer cells and that it inhibits cell proliferation in a dose-dependent manner. Cell cycle effects of topotecan seem to vary with topotecan concentration, with the duration of treatment, and probably also with the type of cells treated. One-hour treatment with topotecan increased the number of lung cancer cells in the S-phase (23). In lung fibroblast cells, treatment with $0.05 \mu \mathrm{M}$ topotecan mainly caused an accumulation of the cells in the S-phase $24 \mathrm{~h}$ after treatment, and subsequently $60 \%$ of the cells were arrested in the G2/M phase (24). In the KU19-20 cells the 6-h treatment with topotecan increased the S-phase fraction, whereas the 48-h treatment increased the G1- and S-phase fractions by amounts dependent on the topotecan concentration.

The combination of survivin-specific siRNA and topotecan reduced cell proliferation and survivin expression synergistically, and the cause of this synergistic effect was examined by investigating the influence of topotecan on survivin expression. Treatment with topotecan alone, however, did not affect survivin expression. The effect of topotecan on survivin expression has been little investigated and the only report of which we are aware is that topotecan decreases the expression of survivin in prostate cancer cells (25), which is inconsistent with our results.

Treatment with topotecan alone did not influence survivin expression, and metachronous administration of survivinspecific siRNA and topotecan did not suppress survivin expression more than did treatment with survivin-specific siRNA alone. Simultaneous administration of survivin-specific siRNA and topotecan, in contrast, suppressed survivin expression synergistically. We therefore hypothesized that topotecan could increase the cellular uptake of siRNA. The results of flow cytometry indicated the cellular uptake of siRNA was increased by the simultaneous administration of topotecan. The cell cycle analysis also revealed that the sub-G1 fraction, which corresponded to apoptotic cells induced by the suppression of survivin, was increased by the simultaneous administration of topotecan. Thus, transfection 
efficiency appeared to be increased by topotecan. Because non-viral vectors have been shown to have a relatively low transfection efficacy (26), numerous studies have explored ways to increase transfection efficiency. According to these studies, transfection efficiency is increased chiefly by improving intracellular transport and/or cellular uptake of vectors. Topoisomerase II inhibitors were shown to enhance the integration of exogenous vectors including liposome by promoting the integration process into human chromosomes (27). Steroids enhance liposome-mediated gene transfer by improving the intracellular transport to the nucleus (28). Insulin has the potential to increase the efficiency of lipofection correlated with the percentage of the S-phase fraction (29). In a mouse model, irradiation was shown to increase the uptake of liposome-DNA complexes in several organs (30). The increased S-phase fraction seen after 6-h treatment with topotecan in the present study may explain the improvement of transfection efficiency. To our knowledge, this is the first report that topotecan can increase transfection efficiency by increasing the cellular uptake of liposome-siRNA complexes.

Survivin also plays an important role in the resistance of carcinomas to chemotherapy and/or radiotherapy (31-34), and the suppression of survivin expression sensitizes cancers to chemotherapy and radiotherapy $(34,35)$. Pennati et al found that ribozyme-mediated inhibition of survivin expression increases the sensitivity of human melanoma cells to topotecan (36). Another mechanism of the synergistic effect of survivinspecific siRNA and topotecan seen in the present study might be sensitization of cancer cells to topotecan by decreased expression of survivin.

In conclusion, the synergistic effect of this combination treatment is due to topotecan increasing the cellular uptake of siRNA. The combination of topotecan and survivin-specific siRNA thus offers an attractive approach to therapy of advanced renal cancer, and topotecan is a useful agent worthy of also being evaluated in the treatment of other types of cancer.

\section{References}

1. Landis SH, Murray T, Bolden S and Wingo PA: Cancer statistics. CA Cancer J Clin 49: 8-31, 1999.

2. Fossa SD: Interferon in metastatic renal cell carcinoma. Semin Oncol 27: 187-193, 2000.

3. Mulders P, Figlin R, De Kernion JB, Wiltrout R, Linehan M, Parkinson D, De Wolf W and Belldegrun A: Renal cell carcinoma: recent progress and future directions. Cancer Res 57: 5189-5195, 1997.

4. Pittman K and Selby P: The management of renal cell carcinoma. Crit Rev Oncol Hematol 16: 181-200, 1994.

5. Mickisch GH: Immunotherapy of renal cell carcinoma. A critical appraisal from a urologist's point of view. Urol Int 63: 16-21, 1999.

6. Pyrhonen S, Salminen E, Ruutu M, Lehtonen T, Nurmi M, Tammela T, Juusela H, Rintala E, Hietanen P and KellokumpuLehtinen PL: Prospective randomized trial of interferon alfa-2a plus vinblastine versus vinblastine alone in patients with advanced renal cell cancer. J Clin Oncol 17: 2859-2867, 1999.

7. Bacoyiannis C, Dimopoulos MA, Kalofonos HP, Nicolaides C, Aravantinos G, Bafaloukos D, Samelis G, Onyenadum A, Kiamouris Ch, Skarlos D, Pavlidis N, Triantafillidis A and Kosmidis P; Hellenic Cooperative Oncology Group: Vinblastine and interferon-gamma combination with and without 13-cis retinoic acid for patients with advanced renal cell carcinoma. results of two phase II clinical trials. Oncology 63: 130-138, 2002.

8. Ambrosini G, Adida C and Altieri DC: A novel anti-apoptosis gene, survivin, expressed in cancer and lymphoma. Nat Med 3: 917-921, 1997.
9. Li F, Ambrosini G, Chu EY, Plescia J, Tognin S, Marchisio PC and Altieri DC: Control of apoptosis and mitotic spindle checkpoint by survivin. Nature 396: 580-584, 1998.

10. Tamm I, Wang Y, Sausville E, Scudiero DA, Vigna N, Oltersdorf T and Reed JC: IAP-family protein survivin inhibits caspase activity and apoptosis induced by Fas (CD95), Bax, caspases, and anticancer drugs. Cancer Res 58: 5315-5320, 1998.

11. La Casse EC, Baird S, Korneluk RG and MacKenzie AE: The inhibitors of apoptosis (IAPs) and their emerging role in cancer. Oncogene 17: 3247-3259, 1998.

12. Ambrosini G, Adida C, Sirugo G and Altieri DC: Induction of apoptosis and inhibition of cell proliferation by survivin gene targeting. J Biol Chem 273: 11177-11182, 1998.

13. Olie RA, Simoes-Wust AP, Baumann B, Leech SH, Fabbro D, Stahel RA and Zangemeister-Wittke U: A novel antisense oligonucleotide targeting survivin expression induces apoptosis and sensitizes lung cancer cells to chemotherapy. Cancer Res 60: 2805-2809, 2000.

14. Kappler M, Bache M, Bartel F, Kotzsch M, Panian M, Wurl P, Blumke K, Schmidt H, Meye A and Taubert H: Knockdown of survivin expression by small interfering RNA reduces the clonogenic survival of human sarcoma cell lines independently of p53. Cancer Gene Ther 11: 186-193, 2004.

15. Sato A, Oya M, Ito K, Mizuno R, Horiguchi Y, Umezawa K, Hayakawa M and Murai M: Survivin associates with cell proliferation in renal cancer cells: Regulation of survivin expression by insulin-like growth factor- 1 , interferon- $\gamma$ and a novel NF-кB inhibitor. Int J Oncol 28: 841-846, 2006.

16. Vindelov LL, Christensen IJ and Nissen NI: A detergent-trypsin method for the preparation of nuclei for flow cytometric DNA analysis. Cytometry 3: 323-327, 1983.

17. Hannon GJ: RNA interference. Nature 11: 244-251, 2002.

18. Sorensen DR, Leirdal M and Sioud M: Gene silencing by systemic delivery of synthetic siRNAs in adult mice. J Mol Biol 327: 761-766, 2003.

19. Ling X, Bernacki RJ, Brattain MG and Li F: Induction of survivin expression by taxol (paclitaxel) is an early event, which is independent of taxol-mediated G2/M arrest. J Biol Chem 279: 15196-15203, 2004.

20. Altieri DC: Cytokinesis, apoptosis and survivin: three for tango? Cell Death Differ 8: 4-5, 2001.

21. Law TM, Ilson DH and Motzer RJ: Phase II trial of topotecan in patients with advanced renal cell carcinoma. Invest New Drugs 12: 143-145, 1994.

22. Ramp U, Mahotka C, Kalinski T, Ebel E, Gabbert HE and Gerharz CD: Topotecan (Hycamtin) responsiveness in human renal carcinoma cell lines of the clear cell and papillary types. Anticancer Res 21: 3509-3517, 2001.

23. Giovannetti E, Mey V, Danesi R, Basolo F, Barachini S, Deri M and Del Tacca M: Interaction between gemcitabine and topotecan in human non-small-cell lung cancer cells: effects on cell survival, cell cycle and pharmacogenetic profile. Br J Cancer 92: 681-689, 2005.

24. Ohneseit PA, Prager D, Kehlbach R and Rodemann HP: Cell cycle effects of topotecan alone and in combination with irradiation. Radiother Oncol 75: 237-245, 2005.

25. Griffith TS and Kemp TJ: The topoisomerase I inhibitor topotecan increases the sensitivity of prostate tumor cells to TRAIL/Apo-2L-induced apoptosis. Cancer Chemother Pharmacol 52: $175-184,2003$.

26. Li S and Huang L: Non-viral gene therapy: promises and challenges. Gene Ther 7: 31-34, 2000.

27. Fujimaki K, Aratani Y, Fujisawa S, Motomura S, Okubo T and Koyama H: DNA topoisomerase II inhibitors enhance random integration of transfected vectors into human chromosomes. Somat Cell Mol Genet 22: 279-290, 1996.

28. Koster F, Felberbaum R, Finas D, Wunsch K, Schulz C, Diedrich $\mathrm{K}$ and Hauser C: Progesterone and estradiol enhance lipid mediated transfection of Sk-Br-3 mammalian cancer cells. Int J Mol Med 9: 617-620, 2002.

29. Ohmiya N, Emi N, Niwa Y, Goto H and Hayakawa T: Insulinenhanced liposome-mediated gene transfer into a gastric carcinoma cell line. Clin Exp Pharmacol Physiol 29: 544-548, 2002.

30. Harrington KJ, Rowlinson-Busza G, Syrigos KN, Uster PS, Vile RG, Peters AM and Stewart JS: The effect of irradiation on the biodistribution of radiolabeled pegylated liposomes. Int J Radiat Oncol Biol Phys 50: 809-820, 2001.

31. Saxena A, Yashar C, Taylor DD and Gercel-Taylor C: Cellular response to chemotherapy and radiation in cervical cancer. Am J Obstet Gynecol 192: 1399-1403, 2005. 
32. Ikeguchi $\mathrm{M}$ and Kaibara N: Changes in survivin messenger RNA level during cisplatin treatment in gastric cancer. Int J Mol Med 8: 661-666, 2001

33. Azuhata T, Scott D, Takamizawa S, Wen J, Davidoff A, Fukuzawa $M$ and Sandler A: The inhibitor of apoptosis protein survivin is associated with high-risk behavior of neuroblastoma. J Pediatr Surg 36: 1785-1791, 2001.

34. Rodel F, Hoffmann J, Distel L, Herrmann M, Noisternig T, Papadopoulos T, Sauer R and Rodel C: Survivin as a radioresistance factor, and prognostic and therapeutic target for radiotherapy in rectal cancer. Cancer Res 65: 4881-4887, 2005.
35. Fuessel S, Herrmann J, Ning S, Kotzsch M, Kraemer K, Schmidt U, Hakenberg OW, Wirth MP and Meye A: Chemosensitization of bladder cancer cells by survivin-directed antisense oligodeoxynucleotides and siRNA. Cancer Lett 232: 243-254, 2006.

36. Pennati M, Binda M, De Cesare M, Pratesi G, Folini M, Citti L, Daidone MG, Zunino F and Zaffaroni N: Ribozyme-mediated down-regulation of survivin expression sensitizes human melanoma cells to topotecan in vitro and in vivo. Carcinogenesis 25: 1129-1136, 2004. 